\title{
Non-Compliant ADaM Datasets Domain
}

National Cancer Institute

\section{Source}

National Cancer Institute. Non-Compliant ADaM Datasets Domain. NCI Thesaurus. Code C111282.

An analysis domain that contains analyses done on non-compliant ADaM datasets. 